\title{
Structured Analysis of Stainless Steel 316L Material of Micro-Forceps Used in Vitrectomy Surgery by Biomechanics Modeling
}

\author{
Ö. KARAÇALI* AND D. ÇETINER \\ Department of Mechanical Engineering, Faculty of Engineering, Istanbul University \\ Avcllar, Istanbul, 34320 Turkey
}

\begin{abstract}
Micro-forceps are a promising minimally invasive means of grasping, peeling, pulling into the eye ball. However, there is currently a diversity of micro-forcep designs and application methods that have, primarily, been intuitively developed by the researchers. To enable the rational design of optimized micro-forcep devices, a greater understanding of human eye biomechanics under small deformations is required. The purpose of this study was to investigate the biomechanical analysis of micro-forceps which are tools for operations that require the microscopic manipulation of tissues in eye surgery conditions. Retinal microsurgery requires extremely delicate manipulation of retinal tissue where tool-to-tissue interaction exists. Behavior of a micro-forcep is analyzed in ANSYS under operation pre-load conditions in vitrectomy. The effect of numerical analysis was determination of strains and stresses in working part of the micro-forcep. Results of analysis indicate diverse values of strains and stresses distribution in working part of the micro-forcep depending on its geometry. With a change in the material, the friction force can be determined using the upper and lower boundaries of different materials to choose from. The results of this analysis are helpful for eye surgeons for clinical interest.
\end{abstract}

DOI: $10.12693 /$ APhysPolA.125.180

PACS: 87.85.gp, 02.70.Dh

\section{Introduction}

The science of biomechanics is the application of mechanical principles on living organisms as tissues, cells, muscles, organs and the motion of them and how their form and function are regulated by basic mechanical properties [1]. To realize this, the finite element method (FEM) [2] is employed which is an advanced computer technique of structural stress analysis and widely accepted as a powerful tool for biomechanics modeling. A finite element model of micro-forcep with accurate geometry [3] and material properties [4] are developed to make realistic investigations on the mechanical behavior of micro-forceps. Micro-forcep tool in the eyeball is shown to depict the insertion and movements of the microsurgery tool and included two types of the forceps as reusable and disposable forceps in Fig. 1.

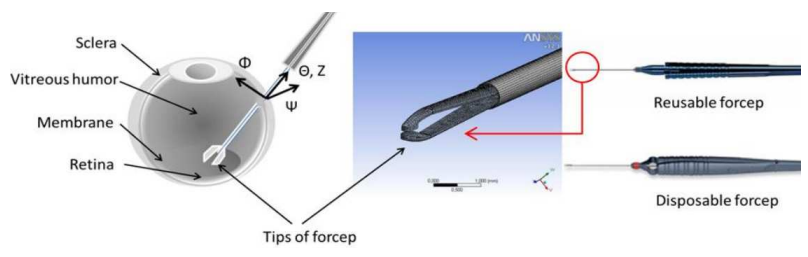

Fig. 1. Micro-forcep tool in the eyeball and tips of the forcep.

*corresponding author; e-mail: ozdogank@istanbul.edu.tr

\section{Micro-forceps in vitrectomy surgery: material and method}

This study analyzes the application of forces resulting from three different movements of micro-forceps examined. As a first move, handle mounted to the main body of micro-forceps by determining the force applied to parts of the closures, while taking place of on-off function. The idea of the force applied to the surgeon's hand in this research provided important information about this surgery tool whether it bothers doctor's hand or not. To obtain this idea as well as the opinions of doctors, and before that, when using the surgical forcep on how much force is applied by the fingers from the analysis of the values of the force, has been applied to the simulation program with this analysis, the ergonomics of the instrument were tested, inadequate materials and/or insufficient mechanism of action have been changed as explained in this research paper. Factors affecting material considerations of vitreoretinal surgery microsurgical tool micro-forceps are given as part of this research in Fig. 2.

Material change, selected by weight of a lighter material can be improved $[4,5]$. But this change, the product should be absolute corrosion resistant due to work environment, which is necessary for the sterilization temperature, durability and strength of the main function of a material that can be made by considering the selection criteria [6]. Similar products on the market were used in stainless steel and titanium. The change of the mechanism of action can be done by extending the length of the connections and increasing the force from the main body parts-mounted handle. By this modification, force trans- 


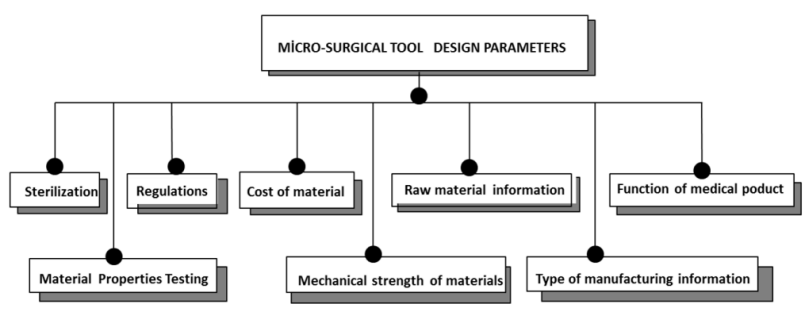

Fig. 2. Factors affecting material considerations of microsurgical tools in biomechanical engineering.

mission links can provide more force by increasing the length of the arm of force. Thus, the main body of the closure of the tip-mounted handle can be achieved by applying less force components. This change may cause enhanced modifications in size of the product, such as parts of the main body mounted on the handle, the length of the channel dimensions of motion area connections.

The second move is a closing act of the end part of the tips in which force transmitted to the links after transferring pieces of the pipe that goes into the pipe mounted. Here, that closing of the orifices of forcep, the end portion of the pushing force was applied by the blades. The minimum required pushing force for the implementation of the closure of two extremes can be found by reducing the force applied to the main body portions of stalk mounted. The thrust force and the tip end portion of the tube exceed the frictional force and the force required for closing is the sum of two blades. Without thrust, the force necessary to turn off the orifices of forcep, after having determined the analysis, thrust force required to turn off the orifices without removing the frictional force can be calculated from the pushing force. Related to compression force applied between the tips, calibrated forceps should have a constant contact surface.

In this study described above, calibrated forceps had variable or "uncontrolled" contact surfaces, despite intentions to incorporate such control for "constant contact surface area" $[7,8]$. One solution to this problem would be to attach a fixed, round, smooth slug to the tip of each arm of the forceps, thus ensuring a constant contact area $[9]$.

With a change in the material, the friction force can be determined using the upper and lower boundaries of different materials to choose from. Here, the pipe material, the end portion, and the end portion of material are more rigid building materials such as spring steel and mild steel structure of a ductile material containing a small amount of carbon that will reduce the force of friction. Lowering to a minimum of frictional force will enable invisible deformations that are degraded to minimum level by time, and will prolong the life of the product.

The third movement, the friction force effect, closing the mouth parts of the tip, as large can be to keep finding function. As a result of a change in the material will be specified by determining the upper and lower force limits. Considering the results of the second movement of the product is long lasting and will help select the most appropriate material: the force required to tip off, tip grip strength, the structure of the micro-forceps body was in the form of general structural analysis. The deformations occurred due to stresses in internal parts of forcep as shown in Fig. 3. Finite element model is employed to analyze these stresses.

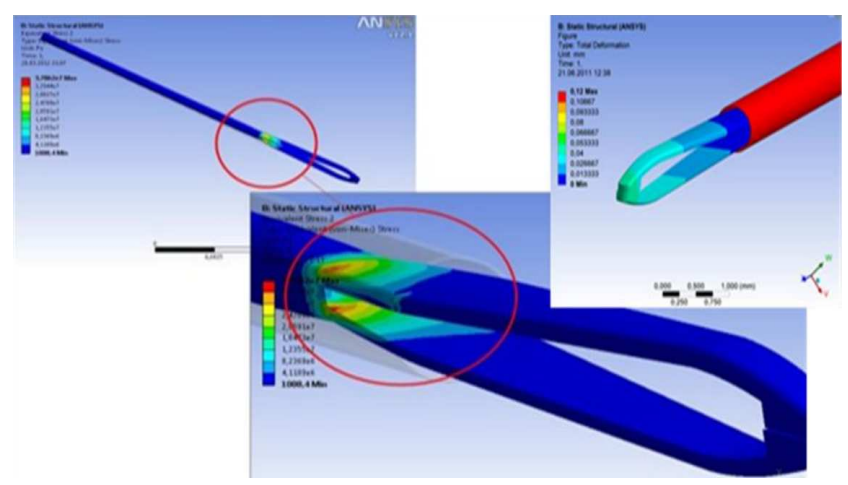

Fig. 3. The deformations occurring due to stresses in internal parts of forcep.

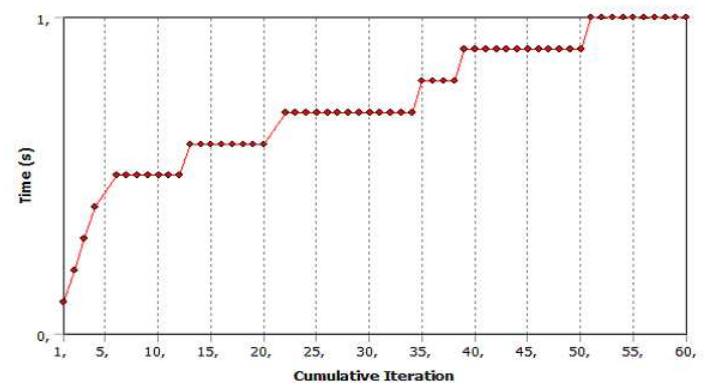

Fig. 4. Time and iteration diagram.

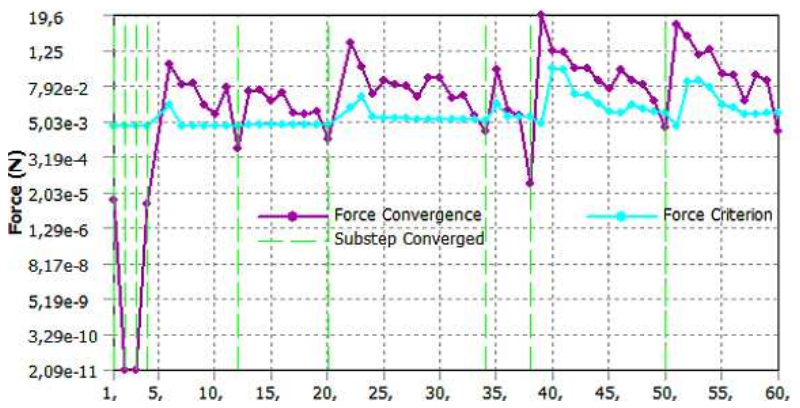

Fig. 5. Convergence diagram.

The material used in the analyzes for micro-forcep is stainless steel 316L. The mechanical properties of the materials used are: elasticity module - $193 \mathrm{GPa}$, Poisson's ratio - 0.3. According to analysis, the time interval for a second of non-linear analysis is completed in a total of 53 iterations as shown in Fig. 4. The movement of the pipe $0.12 \mathrm{~mm}$ and end closure process of iteration and convergence graph are given in Fig. 5. 


\section{Conclusions}

This study has developed a series of predicative finite element analyses of micro-forcep design, which incorporate the forcep, tips of the forcep, retina tissue to model the mechanical characteristics. The relative micro-forcep application force to the surgeon's hand is modeled by analysis of FEM using design parameters in Fig. 2. Experimental in vivo data and FEM results are compared for a specific micro-forcep analysis as given in Fig. 3 .

This model has been used to successfully predict eye retina deformation, at the micron scale, upon application of single micro-forcep. Future studies will use this model to optimize individual micro-forcep design and, following further development of the model, inform the evolution of effective micro-forcep array geometries. This will enable rational design of micro-forcep devices with tailored performance characteristics for specific therapeutic outcomes.

\section{Acknowledgments}

This research is supported by Scientific Research Projects Coordination Unit of Istanbul University, UDP project No. 28023.

\section{References}

[1] H. Grant, S. Settles, IIE Trans. 41, 537 (2009).

[2] ANSYS Manual, Version 14., ANSYS Inc., USA 2012.

[3] M.E. Aguirre, M. Frecker, J. Med. Dev. 2, 015001 (2008).

[4] R.V. Rao, J.P. Davim, Int. J. Adv. Manuf. Technol. 35, 751 (2008).

[5] H. Studer, H. Riedwyl, P. Buchler, Comput. Meth Biomech. Biomed. Eng. 15, 93 (2012).

[6] K. Yang, Y. Ren, Sci. Technol. Adv. Mater. 11, 014105 (2010).

[7] Y. Ida, N. Sugita, T. Ueta, Y. Tamaki, K. Tanimoto, M. Mitsuishi, Int. J. CARS 7, 27 (2012).

[8] I. Iordachita, Z. Sun, M. Balicki, J.U. Kang, S.J. Phee, Int. J. CARS 4, 383 (2009).

[9] A.C. Rau, M. Frecker, A. Mathew, E. Pauli, J. Med. Dev. 5, 41001 (2011).

[10] H. Mater. Med. Dev., Ed. J.R. Davies, ASM Int., Mateerials Park (OH) 2003, Ch. 1, p. 1. 